\title{
Importance of mitigation measures for hospital transmission of SARS-CoV-2 at the onset of the epidemic: the experience of Brescia, Northern Italy
}

\author{
Valentina Marchese ${ }^{1}$ - Beatrice Formenti ${ }^{1}$. Gloria Cola ${ }^{2}$. Natalia Gregori ${ }^{2}$ Elisa Albini ${ }^{3}$. Giuseppe De Palma ${ }^{4}$. \\ Irene Possenti ${ }^{5} \cdot$ Marco Scala $^{6} \cdot$ Francesco Castelli $^{1} \cdot$ Alberto Matteelli $^{1}$
}

Received: 20 March 2021 / Accepted: 1 September 2021 / Published online: 15 September 2021

(c) The Author(s) 2021

\begin{abstract}
Purpose Since the first Italian case of SARS-CoV-2 was detected in Lombardy (Northern Italy) Italy quickly became one of the worst-affected European countries, with a severe impact on health-care workers (HCWs). In the first epidemic, HCWs accounted for $12 \%$ of all national COVID-19 cases. We evaluated the burden of COVID-19 among HCWs and other nonhealth-care workers (nHCWs) in a large Italian hospital.

Methods From March 1st to May 31st 2020, we performed a retrospective study at ASST Civil Hospital, in the Province of Brescia, Lombardy. The study population included all hospital personnel $(n=9265)$, categorized by professional status. Results A SARS-CoV-2 test was performed in 3572 workers (38.5\%), with a positive result in 552 (5.9\% of all hospital personnel). The temporal trend of SARS-CoV-2 cases in hospital staff broadly reflected that in the community, with a great majority of infections occurred during March 2020 (87.7\%). From April onward, a steep decrease of positive cases was observed among hospital personnel, while in the community the decrease was much slower. Medical doctors $(8.9 \%)$ and nurses (8.5\%) were the most affected professional categories with a significantly higher risk of SARS-CoV-2 infection (OR 1.436 and OR 1.410, respectively $p<0.0001)$. HCWs in COVID-19 units presented a significantly higher risk of infection compared to HCWs in non-COVID units $(p<0.001)$.

Conclusion HCWs were severely affected by the COVID-19 epidemic, probably associated with an overwhelming burden of work and lack of preparedness in prevention of nosocomial transmission of the infection. The rapid decrease of COVID19 spread in the hospital, registered before the one in the community, suggests that the adopted preventive measures were effective.
\end{abstract}

Keywords COVID-19 $\cdot$ SARS-CoV-2 $\cdot$ Health-care workers $\cdot$ Hospital infection $\cdot$ Mitigation measures $\cdot$ Nosocomial infection

Alberto Matteelli

alberto.matteelli@unibs.it

1 University Department of Infectious and Tropical Diseases, WHO Collaborating Centre for TB/HIV Collaborative Activities and for TB Elimination Strategy, University of Brescia, ASST Spedali Civili of Brescia, Brescia, Italy

2 Department of Infectious and Tropical Diseases, University of Brescia, Piazza Spedali Civili, 1, 25125 Brescia, Italy

3 Section of Occupational Health, Department of Experimental and Applied Medicine, University of Brescia, Brescia, Italy
4 Section of Public Health and Human Sciences, Department of Medical and Surgical Specialties, Radiological Sciences, and Public Health, University of Brescia, Brescia, Italy

5 Department of Statistical Sciences "Paolo Fortunati", University of Bologna, Bologna, Italy

6 Department of Environmental Health Sciences, Instituto di Ricerche Farmacologiche Mario Negri IRCCS, Milan, Italy 


\section{Background}

On 11 March 2020, CoronaVirus Disease 2019 (COVID19), a new respiratory infectious disease caused by severe acute respiratory syndrome coronavirus 2 (SARS-CoV-2), was declared by WHO as a pandemic [1]. With over 113 million confirmed cases and 2.5 million deaths worldwide as of 1st of March 2021 [2], COVID-19 has posed enormous challenges to health systems globally. The sudden onset of the pandemic found hospitals and health-care workers (HCWs) unprepared and determined the need to convert significant resources in response to the epidemic. HCWs and all hospital personnel faced an increased risk of SARS-CoV-2 infection due to their professional exposure. Shortages of personal protective equipment (PPE) and a delay in the application of strict isolation measures [3-6] were common at this stage. According to WHO data [7], in September 2020 HCWs accounted for $14 \%$ of worldwide COVID-19 cases, reaching 35\% in some countries, while representing less than $3 \%$ of the general population. In the first report of 138 COVID-19-positive patients from Wuhan, China, 29\% of the cases were HCWs [8], while in a cohort study from a single hospital in Spain, $11 \%$ of HCWs had COVID-19 during the first months of the epidemic [9].

Italy registered its first case of SARS-CoV-2 on February 21,2020 , and soon became one of the worst-affected European countries. HCWs accounted for $12 \%$ of all national cases by June 2020 [10].

HCWs represent one of the most precious resources in the fight against the pandemic; focusing research on prevention and control strategies for SARS-CoV-2 nosocomial infection is essential for many reasons. First, infected HCWs and hospital personnel could transmit the virus to vulnerable patients and to close contacts both in hospital and in the community, expanding COVID-19 spread [6]. Moreover, high rates of infection among health personnel inevitably lead to staff shortage weakening the health-care system [11]. This provides the rationale for prioritizing protective interventions among $\mathrm{HCWs}[5,6,11]$.

We studied the impact of SARS-CoV-2 infection among hospital personnel in a tertiary hospital of Brescia, Lombardy (6.3\% of all Italian cases, as on May 31st, 2020) to identify factors associated with a higher risk of SARSCoV-2 nosocomial transmission.

\section{Methods}

\section{Population and study period}

We performed a retrospective analysis of data collected between March 1st and May 31st, 2020, at the Local Health and Social Organization (ASST) Civil Hospital of Brescia, a public tertiary referral University hospital. The hospital provides care to the entire municipality at three different sites: Civile Hospital that is the main city hospital, and the decentralized Montichiari Hospital, and Gardone Val Trompia Hospital. Our study population included all hospital personnel working during the study period, categorized by professional status: non-health-care workers (nHCWs), including personnel without any contact with patients [i.e., pharmacists, engineers, sterilization personnel, drivers, priests, secretaries, food service staff, administrative staff (manager, accountant, human resources office, legal affairs)]; and HCWs, including medical doctors (MDs), residents, nurses and midwives, health-care assistants (HCAs) and technicians (i.e., physiotherapists and X-ray technicians). Students were excluded from the analysis. On May 31st, 2020, there were 9265 workers employed by the ASST Civil Hospital of Brescia, of whom 2497 (26.9\%) were nHCWs and 6,345,768 (68.5\%) were HCWs, including 2481 nurses $(26.8 \%)$ and 1209 HCAs.

\section{Diagnostic method and definition of COVID-19}

According to the hospital protocol [12], during the study period any $\mathrm{nHCW}$ and $\mathrm{HCW}$ was tested for SARS-CoV-2 if presenting with: body temperature $>37.5^{\circ} \mathrm{C}$, fatigue, headache, myalgia and at least one among cough, sore throat and dyspnea, or history of close contact with a confirmed case of COVID-19 (defined by WHO's guidelines [13]). Testing was based on nose-pharyngeal (NP) swabs for detection of SARS-CoV-2 nucleic acid by specific real-time polymerase chain reaction (RT-PCR). A confirmed case of COVID-19 was defined by a swab positive for SARS-CoV-2 RT-PCR. Every SARS-CoV-2 positive result from any accredited laboratory had to be notified to the occupational health services of the hospital.

\section{Variables and data collection}

We retrospectively collected data regarding confirmed COVID-19 cases among hospital employees mining the databases of the Occupational Health Department and Human Resources Department. For each infected case we 
extracted demographic data (age and sex), professional status and site of work. The database was anonymized.

Hospital employees were categorized according to their professional status and place of work during the study period. Units of the main Hospital Civile were divided into non-COVID units and COVID dedicated units $(n=16)$.

\section{Questionnaire-based survey}

To get an insight into the characteristics of COVID-19 spread within the hospital, we performed a voluntary online questionnaire sub-study targeting COVID-19-affected employees. The questionnaire was available from the beginning of June to 31 July 2020 . The survey covered the following areas: (i) symptoms and time of onset; (ii) suspected intraor extra-hospital source of contagion: patients, colleagues, family members, others; (iii) circumstances and location of presumptive contact with the source of contagion: invasive and non-invasive procedures generating droplets, indoor place, use and type of mask, and kind of close contact [14].

\section{Statistical analysis}

Descriptive analysis of the data was performed by determining the incidence rate of SARS-CoV-2 infection in HCWs stratified by professional status and operational unit (OU). Categorical variables were summarized as numbers and percentages and continuous variables were expressed as mean and standard deviation (SD). The outcome was correlated with different categorical variables using the Chi-squared test, with $p<0.05$ taken to indicate significance. Possible associations between variables and the primary outcome were explored by odds ratios (OR). ORs were presented with 95\% CIs, calculated using the normal approximation (Wald). All analyses were performed using SAS version 9.4 (SAS Institute, Cary, NC).

\section{Results}

\section{Incidence of COVID-19 by professional role of hospital employees}

During the study period, 3572 workers (38.5\%) were tested for SARS-CoV-2, of whom 552 had confirmed infection, accounting for $5.9 \%$ of all employees. The mean age of those infected was $46.23(\mathrm{SD} \pm 11.1)$, and $415(70 \%)$ were female. The categories receiving the higher number of tests were nurses $(n=1424 ; 57.3 \%)$, along with HCAs $(n=687 ; 56.8 \%)$ and MDs $(n=594 ; 50.8 \%)$. The highest positivity rates were observed among MDs (8.9\%, 104 positive cases) and nurses $(8.5 \%, 213$ positive cases), followed by technicians $(7.6 \%$, 47 positive cases) and HCAs $(6.9 \%, 84$ positive cases). As expected, nHCWs presented the lower proportion of PCR tests performed ( $n=242,9.7 \%)$, with the lowest incidence rate $(<2 \%)$. Applying a logistic regression model to our data, all the HCWs except for the residents showed a significantly higher risk of being infected by SARS-CoV-2 than nHCWs $(p<0.05)$ (Table 1$)$.

HCWs of Infectious Disease Department, the first department accepting confirmed COVID-19 patients, had a positive-NP swab rate of $25 \%$ with a significantly increased risk of COVID-19 infection compared to other departments (OR 1.864; CI 1.164-2.986; $p$ value 0.0009 ).

Table 1 Proportion of hospital personnel tested for SARS-CoV-2, positivity rate, and proportion positive over the population, according to professional status

\begin{tabular}{|c|c|c|c|c|c|c|}
\hline Professional status & Total, $N(\%)$ & Tested, $N(\%)$ & Positive rate, $N(\%)$ & $\begin{array}{l}\text { Incidence } \\
\text { rate, } \%\end{array}$ & $p$ value & $\mathrm{OR}(\mathrm{CI})$ \\
\hline nHCWs & $2497(26.9)$ & $242(9.7)$ & $46(19)$ & 1.8 & "Ref" & "Ref" \\
\hline MD & $1167(12.6)$ & $594(50.9)$ & $104(17.5)$ & 8.9 & $<0.0001 *$ & $\begin{array}{l}5,430 \\
(3.779-7.802)\end{array}$ \\
\hline Residents & $872(9.4)$ & $323(37)$ & $53(16.4)$ & 6.0 & 0.88 & $\begin{array}{l}3.693 \\
(2.451-5.565)\end{array}$ \\
\hline Nurses & $2481(26.8)$ & $1424(57.4)$ & $213(14.9)$ & 8.5 & $<0.0001 *$ & $\begin{array}{l}5.332 \\
(3.823-7.437)\end{array}$ \\
\hline HCAs & $1209(13)$ & $687(56.8)$ & $84(12.2)$ & 6.9 & $0.04 *$ & $\begin{array}{l}4.490 \\
(3.092-6.520)\end{array}$ \\
\hline Technicians & $616(6.6)$ & $265(43)$ & $47(17.7)$ & 7.6 & $0.05^{*}$ & $\begin{array}{l}4.714 \\
(3.086-7.2)\end{array}$ \\
\hline Unknown & $423(4.6)$ & $40(9.4)$ & $5(12.5)$ & 1.2 & & \\
\hline Total & $9265(100)$ & 3575 (38.6) & $552(15.4)$ & 5.9 & & \\
\hline
\end{tabular}

$O R$ odds ratio, $C I$ confidence interval

$p$ value from $\chi^{2}$ test 
In a second step of the analysis, we gathered HCAs, residents, technicians and workers of unknown status in a unique category called "other status" and we compared it with nHCWs, nurses and doctors. This logistic regression model confirmed a significantly lower proportion of SARS-CoV-2 infection for nHCWs (OR 0.264; CI $0.189-0.370 ; p$ value $<0.0001)$ and an increased proportion of infection among medical doctors (OR 1.436; CI $1.120-1.841 ; p$ value $<0.0001)$ and nurses (OR 1.410; CI $1.151-1.727 ; p$ value $<0.0001$ ) (Table 2).

\section{Temporal trend of COVID-19 infections}

Among hospital employees, the first confirmed case of COVID-19 was diagnosed on March 2. Of all positiveNP swabs detected during the study period $(n=552)$, the great majority $(484,87.7 \%)$ occurred in March, followed by a sharp decrease in April $(59,10.7 \%)$ and May $(9,1.6 \%)$. Monthly incidence rates were $5.2 \%, 0.63 \%$ and $0.1 \%$.

Figure 1 shows the number of confirmed cases among hospital workers per week, along with the total number of cases notified in Brescia during the study period. The peak of the infection was reached earlier, and flattened earlier, among hospital personnel than in the general population.
Table 2 Logistic regression analysis of the probability of a positive test and incidence rate of SARS-CoV-2 in hospital personnel according to occupational exposure

\begin{tabular}{lccllll}
\hline Professional status & Total, $N(\%)$ & Tested, $N(\%)$ & $\begin{array}{l}\text { Positive rate, } N \\
(\% \text { over tested })\end{array}$ & $\begin{array}{l}\text { Incidence } \\
\text { rate, } \%\end{array}$ & $p$ value & OR (CI) \\
\hline Other status & $3120(33.7)$ & $1315(42.1)$ & $189(14.3)$ & 6.0 & "Ref" & "Ref" \\
MD & $1167(12.6)$ & $594(50.9)$ & $104(17.5)$ & 8.9 & $<0.0001$ & 1.436 \\
& & & & & & $(1.120-1.841)$ \\
Nurses & $2481(26.8)$ & $1424(57.4)$ & $213(14.9)$ & 8.5 & $<0.0001$ & 1.410 \\
& & & & & & $(1.151-1.727)$ \\
nHCWs & $2497(26.9)$ & $242(9.7)$ & $46(19)$ & 1.8 & $<0.0001$ & 0.264 \\
& & & & & & $(0.189-0.370)$ \\
\hline
\end{tabular}

$O R$ odds ratio, $C I$ confidence interval $p$ value from $\chi^{2}$ test

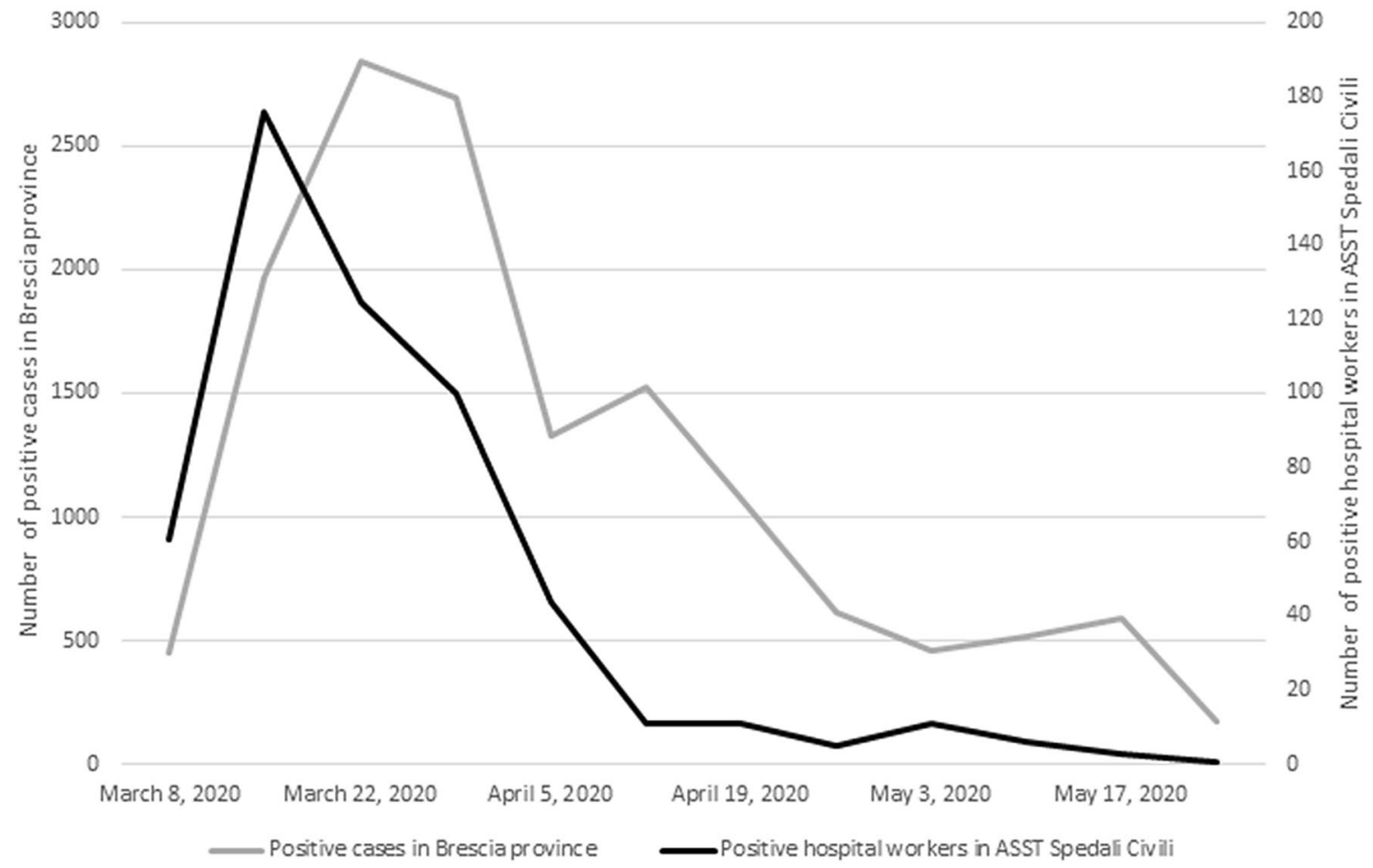

Fig. 1 Weekly cases of SARS-CoV-2 infection among hospital personnel of ASST Spedali Civili and Brescia community 
Among the latter, incidence continued to be high during April and May 2020.

\section{Incidence of SARS-CoV-2 infection among hospital personnel based on departments of exposure}

The analysis of the cumulative incidence of SARS-CoV-2 infection by departments of exposure was performed only for the main hospital personnel (8121 workers, more than $80 \%$ of the ASST staff). Most workers were employed in non-COVID units (6618 staff, $81.4 \%$ vs. $1503,18.5 \%$ ) that recorded a cumulative incidence rate of $4.4 \%$, as opposed to COVID units where the incidence was $11 \%$.

Staff of COVID units had a significantly higher risk of infection compared to staff of non-COVID units (OR 2.74, CI $2.25-3.34, p$ value $<0.001)$.

\section{Perceived routes of COVID-19 transmission through online voluntary questionnaire}

i. Symptoms - Of the 552 infected workers, 343 (62.1\%) completed the questionnaire section regarding symptoms before the diagnosis of COVID-19. The vast majority (72.0\%) of the respondents declared to have had symptoms before or at the moment of the test, such as anosmia, cough, myalgia and fatigue. Overall, $66.8 \%$ had a body temperature above $37^{\circ} \mathrm{C}$. Twentyeight percent of the respondents declared not to have had any symptoms before COVID-19 diagnosis and had been tested because of a contact with a patient with COVID-19.

ii. Presumptive source of transmission-Out of 552 positive workers, 279 (50.5\%) answered regarding the presumptive source of transmission and its modalities. The great majority (88.1\%) assumed to have been infected by SARS-CoV-2 in the hospital setting. Among them, almost $40 \%$ reported a colleague as a possible source of infection, who later was diagnosed with COVID-19; on the other hand, $31.9 \%$ of

Table 3 Presumptive source of transmission

\begin{tabular}{ll}
\hline Presumptive source of contagion & $\begin{array}{l}\text { Positive } \\
\text { interviewees, } \\
N(\%)\end{array}$ \\
\hline Colleague & $110(39.4)$ \\
Patient & $89(31.9)$ \\
Non-invasive procedure & $68(76.4)$ \\
Invasive procedure & $21(23.6)$ \\
Patient and colleague & $47(16.9)$ \\
Out of hospital & $33(11.8)$ \\
Total & 279 \\
\hline
\end{tabular}

respondents perceived to have acquired the infection from hospitalized patients, although most of them (76.4\%) did not report performing invasive investigations (i.e., intubation and aspiration). Nearly 17\% of respondents did not identify the source, since they have had contacts with both infected patients and colleagues (Table 3).

iii. Use of mask at the time of infection-Two hundred and thirteen (38.6\%) of 552 infected employees answered regarding the use of masks at the time of infection. Almost 30\% reported the use of FFP2/3 masks, while surgical masks were reportedly worn by $38.0 \%$ of the respondents. The remainder stated not to have worn any type of mask at the time of the presumptive contagion. More than $75 \%$ of the positive workers who had unprotected contact had also got in touch with colleagues (Table 4).

\section{Discussion}

Brescia was one of the first areas to be devastated by the SARS-CoV-2 epidemic in Europe, with a cumulative incidence of $>500 / 100,000$ as on June 3rd, 2020 [10]. The temporal trend of SARS-CoV-2 cases in the hospital setting broadly reflected that in the community, as the great majority of infections occurred during March 2020 (87.7\%). However, in April 2020, a sharp decrease in the curve was observed in the hospital, while the community continued to be severely affected in April and May [15].

During the first epidemic wave of SARS-CoV-2, hospital personnel in Brescia had an incidence of 5.9\%. In a recent meta-analysis, the estimated prevalence of SARS-CoV-2 infection in HCWs was $11 \%$, although high variability was detected among the evaluated studies (from 0.4 to 57\%) [16]. The inclusion of nHCWs in our study may explain the lower incidence seen compared to others $[6,9]$. Since the

Table 4 Use of PPE among infected interviewees

\begin{tabular}{ll}
\hline Mask & $\begin{array}{l}\text { Positive } \\
\text { interviewees, } \\
N(\%)\end{array}$ \\
\hline FFP2/3 mask & $61(28.6)$ \\
Surgical mask & $81(38.0)$ \\
No mask & $71(33.4)$ \\
Contact with patient & $12(16.9)$ \\
Contact with colleague & $54(76.0)$ \\
Unknown & $5(7.1)$ \\
Total & 213 \\
\hline
\end{tabular}


beginning of the epidemic, home-working strategies have been implemented to reduce the risk of transmission. This intuitively contributed to the lower incidence detected in nHCWs $(p<0.05)$. Moreover, hospital directives released on 5th March 2020 did not indicate the need of PPE for hospital personnel not directly involved in assistance to COVID patients, following the indication of the World Health Organization at that time $[17,18]$. Nevertheless, the hospital offered surgical masks to both HCWs and nHCWs, and FFP2/FFP3 in case of direct assistance to COVID patients. Universally, PPE utilization was progressively introduced on 23rd March, although not mandatory but highly suggested, and became required for everybody on the 18th of May [19, 20]. Interestingly, nHCWs were much less tested compared to HCWs, but had a slightly higher positivity rate (19\% vs $15.2 \%)$. This might be explained by the fact that nHCWs were a lower priority for PPE distribution (PPE not indicated unless in case of front-office activities until the 23rd March) and nHCWs might also have had a lower perception of the risk. HCWs, feared of being a possible source of infection, self-established very early quarantine measures, limiting contact with relatives and friends far beyond this became mandatory for the general population. This attitude may have reduced unprotected exposure to unknown infected people in community settings, especially in the early epidemic. The impact of in-hospital versus community-acquired infection is still to be well understood, and some authors highlighted the possibility of community transmission for HCWs too [16]. However, the importance of workplace exposure had already been suggested by other studies [16]. In our survey, hospital staff had a significantly greater incidence compared to the community (5.9\% versus $1.1 \%)$. Medical doctors (8.9\%) and nurses $(8.5 \%)$ were at higher risk of being affected by SARS$\mathrm{CoV}-2$ compared to other professional categories. Moreover, personnel involved in COVID dedicated units had more than double the risk of being infected with SARS-CoV-2 compared to those working in non-COVID units (OR 2.74). This is in contrast with studies performed in Wuhan at the beginning of the epidemic which showed a higher risk of infection in low-risk units, where workers were less trained on the use of PPE $[6,21]$. Conversely, more recent Italian [22-24] and American studies [25] showed results similar to ours, alongside the Denmark study on seroprevalence (thus identifying also asymptomatic cases) that demonstrated a higher prevalence for workers in COVID-19 units than other frontline workers [26]. Besides, another retrospective survey conducted in our hospital found no correlation between working in COVID units and positive serostatus, presumably due to a higher risk awareness [27].

At the very beginning of the epidemic, the time investigated by our study, transmission routes of SARS-CoV-2 had not been fully elucidated and risk mitigation measures (i.e., specific distinction of departments in COVID-19 units and non-COVID-19 units, proper use of PPE) had not been established yet. These factors facilitated in-hospital transmission in our Infectious Diseases Department, the first one accepting confirmed and suspected COVID-19 patients.

Conversely, the steep decrease of positive cases among hospital personnel observed after March probably reflects adequate preventive and tracing nosocomial measures implemented on daily bases, while new scientific evidence was cumulating [21, 28-31].

Finally, the perception of those who answered the questionnaire suggests that infections were mainly acquired from the working environment ( $88 \%$ of 279 positive interviews), where at least $40 \%$ of cases were reportedly acquired by contact with colleagues. The fact that about one-third of 213 interviewees reported not to have worn any masks at the time of presumptive contagion supports the important role of masks in preventing infection spread [28-31].

The role of asymptomatic and pre-symptomatic infections in transmission was debated at the time of the investigation. More recent evidence supports the role of asymptomatic cases in the transmission of the infection [32, 33]. In this scenario, risk mitigation measures adopted in hospital settings have certainly reduced transmission from asymptomatic or pre-symptomatic affected people. The risk of asymptomatic transmission was unknown in the early epidemic, and this could have determined a false perception of safety among employees when interacting with each other, especially in the few and short breaks during highly stressful shifts.

Our study has limitations. First, its retrospective design limited the number of variables in this study. Second, we did not detect asymptomatic infections and the real infection rate in the whole cohort might have been underestimated. Third, the results of the questionnaire analysis may have been biased by individual beliefs and perceptions on the time and site of exposure. Finally, the findings of the questionnaire may be biased by the proportion (about 50 of non-respondents).

\section{Conclusion}

We report evidence of great risk of acquisition of SARSCoV-2 among health-care workers at the very beginning of the epidemic. The infection spread very quickly among the clinical personnel before mitigation measures were recommended and implemented. However, the early flattening of the curve of infection of in-hospital staff, compared to the general population, suggests that preventive interventions were effective. As expected, health-care workers of COVID dedicated units presented a significantly higher risk of contracting SARS-CoV-2. Our data show that the adoption of 
preventive measures together with a supply of adequate PPE played a pivotal role in containing hospital transmission.

The SARS-CoV-2 epidemic of 2019 warns us about the risk for health personnel at the onset of epidemics due to new agents. Effective preparedness policies should always be rapidly implemented to protect people and places of care.

Author contributions Conceptualization: VM, BF, GC and AM. Data curation: EA, GDP. Formal analysis: IP, MS. Supervision: FC, AM. Writing original draft: VM, BF, GC, NG and AM. Writing review and editing: VM, BF, GC, NG, EA, GDP, IP, MS, FC, AM.

Funding Open access funding provided by Università degli Studi di Brescia within the CRUI-CARE Agreement.

\section{Declarations}

Conflict of interest We have read and understood the Infection policy on declaration of interests and have no relevant interests to declare.

Open Access This article is licensed under a Creative Commons Attribution 4.0 International License, which permits use, sharing, adaptation, distribution and reproduction in any medium or format, as long as you give appropriate credit to the original author(s) and the source, provide a link to the Creative Commons licence, and indicate if changes were made. The images or other third party material in this article are included in the article's Creative Commons licence, unless indicated otherwise in a credit line to the material. If material is not included in the article's Creative Commons licence and your intended use is not permitted by statutory regulation or exceeds the permitted use, you will need to obtain permission directly from the copyright holder. To view a copy of this licence, visit http://creativecommons.org/licenses/by/4.0/.

\section{References}

1. WHO. Timeline of WHO's response to COVID-19. 2020. www. who.int/emergencies/diseases/novel-coronavirus-2019/interactivetimeline.

2. WHO Weekly epidemiological update. Accessed 2 Mar 2021.

3. WHO. Health workforce policy and management in the context of the COVID-19 pandemic response. Accessed 3 Dec 2020.

4. Lancet T. COVID-19: Protecting health-care workers. Lancet. 2020;395:922.

5. Sikkema RS, Pas SD, et al. COVID-19 in health-care workers in three hospitals in the south of the Netherlands: a cross sectional study. Lancet Infect Dis. 2020;20:1273-80.

6. Wei J-T, Liu Z-D, et al. Epidemiology of and risk factors for COVID19 infection among health care workers: a multi-centre comparative study. Int J Environ Res Public Health. 2020;17:7149. https://doi. org/10.3390/ijerph17197149.

7. WHO, Keep health workers safe to keep patients safe. 2020. https:// www.who.int/news/item/17-09-2020-keep-health-workers-safe-tokeep-patients-safe-who.

8. Wang D, Hu B, Hu C, Zhu F, Liu X, Zhang J, et al. Clinical characteristics of 138 hospitalized patients with 2019 novel coronavirusinfected pneumonia in Wuhan, China. JAMA. 2020;323:1061e9.

9. Suárez-García I, de Martínez Aramayona López MJ, Sáez Vicente A, Lobo Abascal P. . SARS-CoV-2 infection among healthcare workers in a hospital in Madrid, Spain. J Hosp Infect. 2020;106:357-63. https://doi.org/10.1016/j.jhin.2020.07.020.

10. ISS, Sorveglianza integrata COVID-19 in Italia, Aggiornamento nazionale 3 Giugno. 2020.
11. Barranco R, Ventura F. COVID-19 and infection in health-care workers: an emerging problem. Med Leg J. 2020;88:65-6.

12. ASST Spedali Civili di Brescia, Procedures for contact case management in workers-version March 2, 2020.

13. World Health Organization (WHO). Global Surveillance for human infection with coronavirus disease (COVID-19) virus. Interim Guid. 2020;(February):27-29. https://www.who.int/publicationsdetail/global-surveillance-for-human-infection-with-novel-coron avirus-(2019-ncov).

14. World Health Organization. 2020. Global surveillance for COVID19 caused by human infection with COVID-19 virus: interim guidance, 20 March 2020. World Health Organization. https://apps.who. int/iris/handle/10665/331506. License: CC BY-NC-SA 3.0 IGO.

15. Statistiche coronavirus Brescia. Published 2020. Accessed 20 Nov 2020. https://statistichecoronavirus.it//coronavirus-italia/coronavirus-lombardia/coronavirus-brescia/.

16. Gómez-Ochoa SA, Franco OH, Rojas LZ, Raguindin PF, Roa-Díaz ZM, Wyssmann BM, Guevara SLR, Echeverría LE, Glisic M, Muka T. COVID-19 in health-care workers: A living systematic review and meta-analysis of prevalence, risk factors, clinical characteristics, and outcomes. Am J Epidemiol. 2021;190:161-75. https://doi.org/ 10.1093/aje/kwaa191 (Erratum in: Am J Epidemiol. 2021 Jan 4;190(1):187. PMID: 32870978; PMCID: PMC7499478).

17. World Health Organization. (2020). Rational use of personal protective equipment for coronavirus disease (COVID-19): interim guidance, 27 February 2020. World Health Organization. https:// apps.who.int/iris/handle/10665/331215. License: CC BY-NC-SA 3.0 IGO.

18. ASST Spedali Civili of Brescia, Unità di Crisi COVID-19, internal directive on PPE, updated on 5th of March 2020.

19. ASST Spedali Civili of Brescia, Unità di Crisi COVID-19, internal directive on PPE, updated on 23rd of March 2020.

20. ASST Spedali Civili of Brescia, Unità di Crisi COVID-19, internal directive on PPE, updated on 18th of May 2020.

21. Zheng L, Wang X, Zhou C, et al. Analysis of the infection status of healthcare workers in Wuhan during the COVID-19 outbreak: a cross-sectional study. Clin Infect Dis. 2020;71:15. https://doi.org/ 10.1093/cid/ciaa588.

22. Fusco FM, Pisaturo M, et al. COVID-19 among healthcare workers in a specialist infectious diseases setting in Naples, Southern Italy: results of a cross-sectional surveillance study. J Hosp Infect. 2020;105:596-600.

23. Felice C, Di Tanna GL, et al. Impact of COVID-19 outbreak on healthcare workers in Italy: Results from a national e-survey. J Community Health. 2020;46:675-83.

24. Bellizzi S, Fiamma M, et al. COVID-19: The daunting experience of healthcare workers in Sardinia. Italy. Infect Control Hosp Epidemiol. 2020. https://doi.org/10.1017/ice.2020.149.

25. Misra-Hebert, et al. Impact of COVID-19 on healthcare workers. J Gen Intern Med. 2020;35:3293-301.

26. Iversen K, Bundgaard H, Hasselbalch RB, et al. Risk of COVID-19 in health-care workers in Denmark: an observational cohort study. Lancet Infect Dis. 2020;20:1401-8.

27. Comelli A, Focà E, Sansone E, Tomasi C, Albini E, Quiros-Roldan E, Tomasoni LR, Sala E, Bonfanti C, Caccuri F, Caruso A, De Palma G, Castelli F. Serological response to SARS-CoV-2 in health care workers employed in a large tertiary hospital in Lombardy, Northern Italy. Microorganisms. 2021;9:488. https://doi.org/10.3390/micro organisms9030488 (PMID: 33669151; PMCID: PMC7996483).

28. Jin YH, Huang Q, Wang YY, et al. Perceived infection transmission routes, infection control practices, psychosocial changes, and management of COVID-19 infected healthcare workers in a tertiary acute care hospital in Wuhan: A cross-sectional survey. Mil Med Res. 2020. https://doi.org/10.1186/s40779-020-00254-8. 
29. Tong $X$, et al. Surveillance of SARS CoV2 infection among frontline health care workers in Wuhan during COVID-19 outbreak. Immun Inflamm Dis. 2020;8:840-3.

30. Zhan M. Death from COVID-19 of 23 health care workers in China. NEJM. 2020. https://doi.org/10.1056/NEJMc2005696.

31. Chou R, Dana T, et al. Epidemiology of and risk factors for coronavirus infection in health care workers. Ann Intern Med. 2020;. https://doi.org/10.7326/L20-0768.

32. Wilmes P, Zimmer J, Schulz J, et al. SARS-CoV-2 transmission risk from asymptomatic carriers: Results from a mass screening programme in Luxembourg. Lancet Reg Health Eur. 2021;4:100056. https://doi.org/10.1016/j.lanepe.2021.100056 (Epub 2021 Feb 27. PMID: 33997830; PMCID: PMC7912359).

33. Rivett L, Sridhar S, Sparkes D, et al. Screening of healthcare workers for SARS-CoV-2 highlights the role of asymptomatic carriage in COVID-19 transmission. Elife. 2020;9: e58728. https:// doi.org/10.7554/eLife.58728 (Published 2020 May 11). 